
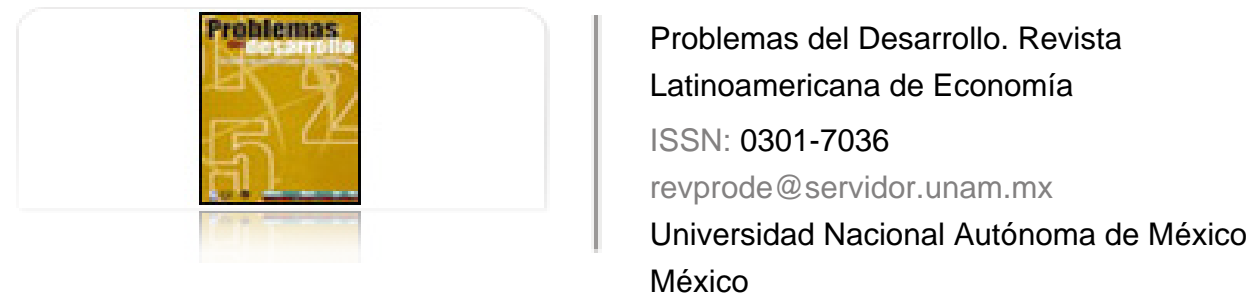

Aguado Quintero, Luis Fernando; Girón Cruz, Luis Eduardo; Salazar Silva, Fernando Una aproximación empírica a la relación entre educación y pobreza

Problemas del Desarrollo. Revista Latinoamericana de Economía, vol. 38, núm. 149, abril-junio, 2007, pp. 35-60

Universidad Nacional Autónoma de México

Distrito Federal, México

Disponible en: http://www.redalyc.org/articulo.oa?id=11820124003

Cómo citar el artículo

- Número completo

- Más información del artículo

Página de la revista en redalyc.org

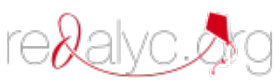

Sistema de Información Científica

Red de Revistas Científicas de América Latina, el Caribe, España y Portugal Proyecto académico sin fines de lucro, desarrollado bajo la iniciativa de acceso abierto 


\section{UNA APROXIMACIÓN EMPÍRICA A LA RELACIÓN ENTRE EDUCACIÓN Y POBREZA*}

\section{Luis Fernando Aguado Quintero** Luis Eduardo Girón Cruz*** Fernando Salazar Silva****}

Fecha de recepción: 16 de marzo de 2006. Fecha de aceptación: 12 de noviembre de 2006.

\section{Resumen}

Al emplear un modelo Logit de elección múltiple ordenado y modelos de ecuaciones estructurales, se encuentra una clara relación entre educación y pobreza; los estructurales revelan la retroalimentación entre ambas variables. Producto de esta última observación, se plantea que aunque la inversión en capital humano mediante la educación es un factor clave para mejorar el ingreso de las personas, ésta por sí sola no es suficiente para alterar el cambio de condición de pobre a no pobre, pues se requiere un ambiente macroeconómico favorable en el cual individuos pobres educados en el mercado de trabajo incrementen su flujo de ingresos.

Palabras claves: pobreza, educación, crecimiento económico, modelos estructurales, modelos Logit de elección múltiple.

* Este documento es parte de un proyecto de investigación denominado "Educación y Po breza en el Valle del Cauca", financiado por la Coordinación Institucional de Investigaciones de la Pontificia Universidad Javeriana en Cali, Colombia. Se agradece la colaboración en el procesamiento de la información de Alexander Alegría, además de Carolina Gómez, Daniella Uribe y David Mejía.

* Profesor asistente, Departamento de Economía, integrante del Grupo de Investigación en Desarrollo Regional (GIDR), Pontificia Universidad Javeriana, Cali, Colombia. Correo electrónico:1faguado@puj.edu.co

*** Profesor asistente, Departamento de Economía, integrante del Grupo de Investigación en Desarrollo Regional (GIDR), Pontificia Universidad Javeriana, Cali, Colombia. Correo electrónico: legiron@puj.edu.co

**** Profesor asistente, Departamento de Economía, integrante del Grupo de Investigación en Desarrollo Regional (GIDR), Pontificia Universidad Javeriana, Cali, Colombia. Correo electrónico: fsalazar@puj.edu.co 
Abstract

Using an ordered Logit multiple choice model and structural equations models, we find a clear relationship between education and poverty. The structural equation models reveal a feedback between both variables. Given this observation, the study proposes that although investment in human capital through education is a key factor for improving people's incomes, on its own it is insufficient for changing conditions from poor to not poor, because it requires a favorable macroeconomic climate in which the educated poor in the labor market increase their income flows.

Key words: poverty, education, economic growth, structural models, Logit multiple choice model.

\section{Résumé}

En employant un modèle Logit de choix multiples ordonnés et des modèles structuraux d'équations, on note une relation évidente entre instruction et pauvreté; les modèles structuraux révèlent l'interaction entre ces deux variables. À partir de cette dernière observation, il est ici soutenu que bien que l'investissement en capital humain via l'éducation soit un facteur déterminant de l'angmentation du revenu des individus, l'instruction ne peut suffire à elle seule à les faire passer de la condition de pauvre à non pauvre, un environnement macroéconomique favorable étant par ailleurs nécessaire pour que des pauvres instruits placés sur le marché du travail puissent augmenter le flux de leurs revenus.

Mots clés: pauvreté, instruction, croissance économique, modèles structuraux, modèles Logit de choix multiples.

\section{Resumo}

Empregando um modelo Logit ordenado de múltipla escolha e modelos de equações estruturais, encontra-se uma clara relação entre educação e pobreza; os estruturais revelam a retro-alimentação entre as duas variáveis. Produto desta última observação, é que a pesar de que a inversão em capital humano por meio da educação é um fator chave para melhorar o ingresso das pessoas, esta por si só não é suficiente para alterar a mudança de condição de pobre a não pobre,já que se requer um ambiente macroeconômico favorável em que os indivíduos pobres educados no mercado de trabalho incrementem seu fluxo de ingressos.

Palavras-chave: pobreza, educação, crescimento econômico, modelos estruturais, modelos Logit de múltipla escolha. 


\section{Introducción}

lgunos estudios empíricos de académicos y entidades multilaterales muestran
una relación entre educación y pobreza, pero teóricamente aún no se ha esta-
blecido un modelo que nos demuestre qué variable afecta a cuál y mucho menos de qué forma, lo que resulta necesario para el desarrollo de una acertada política social.

Si el grado de pobreza depende decrecientemente del nivel educativo, entonces se requiere de una política que lo incremente en los sectores más necesitados de la sociedad. Por el contrario, si el nivel educativo está supeditado de manera decreciente al de pobreza, entonces se precisa una política social que atenúe dicha situación.

Finalmente, si la relación de causalidad implica retroalimentación entre los niveles de pobreza y de educación —es decir, se es pobre por detentar bajos grados de educación y viceversa—, el diseño de la política social debería considerar de forma conjunta la dinámica educación-pobreza.

Puesto que existen 220 millones de habitantes (CEPAL, 2005) en situación de pobreza en Latinoamérica, se requiere que los gobiernos delineen políticas sociales para reducir la situación, uno de los Objetivos de Desarrollo del Milenio (onU, 2000). Más aún, debe ser atendida no sólo por razones éticas y de justicia social, sino también porque genera inestabilidad social, económica y política en las naciones.

Además de proponernos determinar estadísticamente la dirección de la relación entre educación y pobreza en el Valle del Cauca, Colombia, en el nivel urbano, a partir de la teoría del capital humano, se pretende identificar el proceso con el cual los beneficios de la educación contribuyen a la superación de las condiciones de pobreza.

El trabajo se divide en tres partes; en la primera, se presenta el marco de referencia para la relación educación-pobreza. En la segunda, se sugiere y estima un modelo Logit de elección múltiple ordenado y uno de ecuaciones estructurales para dar cuenta de la relación de causalidad entre educación y pobreza; de forma previa, se realiza un análisis descriptivo para comprender esa relación. En la tercera parte, se pretende reconocer el proceso en el que operan los beneficios de la educación para que un individuo transite de un estado pobre a uno no pobre. Por último, se presentan las conclusiones y recomendaciones más relevantes del estudio. 


\section{Marco de referencia}

\section{Pobreza y educación}

A pesar de que se registran millones de personas en todo el mundo en estado de pobreza, no existe unanimidad en la comunidad académica acerca de una definición, sin embargo existen tres enfoques para estudiarla y comprenderla en diferentes sociedades y durante el transcurso del tiempo.

El primero se fundamenta en el ingreso, el consumo y, hasta cierto punto, en el bienestar humano; es ampliamente aceptado por los economistas para entender y medir la condición de pobreza y bienestar de las personas. Por consiguiente, una persona es pobre si sus ingresos no le permiten adquirir los bienes de la canasta básica familiar.

El segundo se concibe como una situación de incapacidad que experimenta un individuo para adquirir bienes que se consideran primarios, como son la educación o la salud, para alcanzar un nivel básico de bienestar humano.

El tercero, compartido especialmente por sociólogos y antropólogos, se concentra en los factores sociales, de comportamiento y políticos del bienestar humano.

La implantación de cada método para medir la pobreza, además de requerir decisiones metodológicas previas sobre la elección de la unidad de análisis y la variable de bienestar, precisa de dos etapas: $a$ ) la identificación (¿cuáles individuos son pobres y qué tan pobres son?) y $b$ ) la agregación (¿cuántos pobres hay?). Más aún, la medida que se utilice para cuantificarla debe satisfacer una serie de propiedades deseables (Ravallion, 1992). No obstante, es esencial la definición de pobreza que se apropie, debido a que de ésta dependerá el tipo de medición de la misma. De acuerdo con diferentes concepciones, en este trabajo se parte desde el punto de vista del ingreso; en ese sentido, se consideran pobres aquellos individuos residentes en hogares cuyos recursos per capita sean inferiores al valor de una línea de pobreza.

Según Ravallion (1998), un hogar cuyas preferencias se pueden representar por la siguiente función de utilidad $u=u(q, x)$, la de gasto del consumidor es $e=e(p, x, u)$, que se interpreta como el costo mínimo de un nivel de utilidad $u$ para un hogar con características $x$ cuando se enfrenta a un vector de precios $p$, y $q$ representa las canastas de bienes que consume el hogar; $e=e(p, x, u)$ cuando se evalúa en el nivel de utilidad real, se corresponde al gasto total real en consumo $y=p^{*} q$, para un hogar que aumenta su utilidad. Si tomamos a $u_{z}$ como nivel de utilidad de referencia necesario para escapar de la pobreza, la línea de ésta sería igual a: $z=e\left(p, x, u_{z}\right)$. 
Lo cual se interpreta así: la línea de pobreza es el costo del nivel mínimo de utilidad para escapar de la pobreza a los precios corrientes y dadas las características personales del hogar. Esta ecuación relaciona cómo ir de la pobreza en términos de utilidad a la pobreza en términos de dinero, pero no cómo definir el nivel de utilidad de la pobreza (Ravallion, 1998:3).

Si bien es cierto que esta concepción tiene una serie de limitaciones en la medida en que sólo considera el ingreso, éste se constituye en una de las variables más importantes para identificar a los pobres.

Ahora bien, la educación, definida como proceso social orientado a la inclusión crítica y ética de los individuos en la cultura, de carácter continuo y que se desarrolla a lo largo de la vida de formas distintas, se considera un derecho fundamental en la sociedad, ${ }^{1}$ por lo cual las políticas públicas deben estar dirigidas a asegurar el acceso y logro de determinados niveles educativos, que permitan desarrollar conocimientos y habilidades a los seres humanos.

Por otro lado, en este trabajo se pone énfasis en la importancia que tiene la educación en el desarrollo integral de la sociedad, particularmente en cuanto a lo económico y social, pues los canales mediante los cuales la educación genera prosperidad y bienestar para la sociedad son relativamente fáciles de distinguir, además de estar bien identificados en la teoría económica.

Hoy es evidente cómo el mundo se encuentra dividido por el grado de desarrollo tecnológico y científico de las naciones; por esa razón, para el departamento del Valle del Cauca resulta políticamente imprescindible prestar atención a la educación como factor para lograr mayor desarrollo económico y social.

\section{La teoría del capital humano ${ }^{2}$}

Si la noción de pobreza se establece en función del ingreso, pobres son quienes tienen ingresos inferiores a un umbral de pobreza. Luego, no poseen los suficientes ingre-

Artículo 26, Declaración Universal de los Derechos Humanos, 1948.

2 No existe una explicación única para el concepto capital humano, aunque frecuentemente se identifica con educación. Estudios recientes plantean la necesidad de redefinir el término en relación con la adquisición. Las nuevas explicaciones - sustentadas en lo anterirorincluyen diversos elementos, algunos de ellos ya recogidos anteriormente en la literatura. De esa forma, se considera que el capital humano puede tener un origen innato o adquirido: a) el innato comprende aptitudes de tipo físico e intelectual, que pueden verse modificadas debido a las condiciones de alimentación y salud. b) El adquirido se irá constituyendo a lo largo de la vida de los sujetos por medio de la educación formal, de la informal y de la expe riencia acumulada. Esos tres tipos de formación adquirida van a condicionar la instrucción 
sos monetarios y subsisten con un mínimo de condiciones de vida socialmente aceptadas, que por lo general son establecidas a partir de requerimientos nutricionales y otros bienes esenciales. Ello sugiere diseñar políticas que promuevan mejoras en los niveles de ingreso para situarlas por encima de dicha línea.

La teoría del capital humano plantea que un aumento en la educación genera efectos positivos en los ingresos de los individuos; ésta incrementa la productividad de un trabajador y eleva los salarios (Becker, 1962; Dahlin, 2003:17; Borjas, 1999:249). En el nivel agregado, la educación también afecta positivamente el crecimiento del producto y la difusión de la tecnología (Barro, 1991; Dahlin, 2003; Sala-i-Martin, 2001).

En resumen, el argumento principal de la teoría del capital humano es una alta correlación causal entre educación, productividad y salario. La experiencia colombiana muestra que existe relación entre educación, formación de capital humano y mayores salarios y productividad (González, Guzmán y Pachón, 1998; Sánchez, Rodríguez y Núñez, 1996; Sánchez y Núñez, 1998; Chávez y Arias, 2002). Para Núñez y Espinosa (2005:6), entre las variables que mejor explican las diferencias en el ingreso per capita está la educación.

Pardo (2006:21) encuentra para Colombia que en el largo plazo un incremento de $1 \%$ del PIB en el gasto público en educación aumentaría la tasa de crecimiento anual de la economía en cerca de $0.14 \%$.

Becker (1964), en relación con el capital humano, propone que la productividad de una persona es el resultado de los conocimientos que se apropie y que ésta precisa su cota de ingreso en el mercado laboral. Dichos conocimientos generales elevan la productividad del trabajador y se adquieren en las instituciones educativas. En oposición, se hallan los conocimientos específicos, los cuales son eficaces para un empleador particular (Johnes, 1993), argumento que emplean las empresas con el objeto de financiar ese tipo de capital humano.

No obstante, en estudios empíricos recientes en el ámbito internacional se encuentra que no hay correlación significativa entre la formación media de la población y el grado de productividad (Easterly, 2003:69-82; De la Fuente, 2004:7-8).

En el plano social y político es ampliamente aceptado en organismos multilaterales (Naciones Unidas, Banco Mundial, Comisión Económica para América Latina) que

laboral y el sistema de valores de los sujetos, que determinarán, junto con las aptitudes innatas, su rendimiento en el trabajo (Giménez, 2005). Para el presente trabajo se entenderá capital humano como lo adquirido mediante la educación formal y la experiencia laboral. 
los beneficios sociales y económicos de la educación se asocian a "las opciones para tener acceso al trabajo, al cuidado de la salud y nutrición, a la consecución de ingresos necesarios para asegurar el bienestar de las personas, y capacitación para un mejor ejercicio de los derechos civiles y políticos" (CEPAL-UNESCO, 2005:12).

Igualmente, se identifica la educación como área prioritaria para el desarrollo humano:

La educación consolida varias dimensiones del desarrollo humano: como capital humano, es una medida de la inversión que la sociedad hace en las personas, como factor de producción, el principal motor de desarrollo al crear conocimiento para mejorar la calidad de vida y favorecer procesos continuos de innovación y como aumento de las capacidades humanas permite que las personas absorban conocimiento, accedan y disfruten de los bienes de la cultura universal (DNP, PNUD, PNDH y GTZ, 2006:7).

De manera adicional, en países en desarrollo, la educación es un sector que puede combatir la reducción de la pobreza y mejorar la equidad, "La inversión en capital educativo es un factor esencial para la reducción de la pobreza y la desigualdad, sobre todo por su capacidad de contribuir a la movilidad social y a la ruptura de la transmisión intergeneracional de la privación" (CEPAL, 2005:77).

Núñez, Ramírez y Cuesta (2005), en un estudio sobre los determinantes de la pobreza en Colombia, en el periodo 1996-2004, encontraron que el tamaño de los hogares y el grado de educación eran determinantes para la pobreza. En ese sentido, los autores sugieren la necesidad de fomentar el acceso a la educación de los más pobres y, en particular, en el nivel superior, en el cual se pueden alcanzar los mayores retornos a la educación.

Ramos (2000) señala la importancia de la relación entre el grado educacional de las personas y los distintos niveles de pobreza presentes en Perú. El trabajo encuentra que la población en edad escolar de hogares pobres reproduce las mismas condiciones de pobreza en las que viven los padres, lo cual trae como consecuencia un círculo vicioso. Las razones esgrimidas para tal situación giran alrededor del bajo nivel de educación de los padres y el menor nivel de ingresos de los hogares, este último asignado a la poca escolaridad del jefe del hogar.

Millán (2005) muestra que en esa región y en el país los pobres tienen muy poco acceso a la educación superior, de tal forma que un resultado de su bajo nivel en ese ámbito sea la gran amplitud de las brechas salariales entre los no pobres y los pobres.

Para Perú, Yalta (1999) utiliza un modelo Probit con el propósito de medir el impacto de la educación sobre la probabilidad de ser pobre, además, consciente de 
que dicha relación se da fundamentalmente en el mercado laboral, también calcula las tasas de retorno de la educación.

Los resultados de Yalta (1999) suponen que la probabilidad de ser pobre es cada vez más baja en la medida en que el nivel educativo se incrementa. Dicha probabilidad pasa de $44.3 \%$, cuando no se tiene ningún nivel educativo, a $9.2 \%$ cuando se tiene educación superior. Por otro lado, señala que el mercado laboral no paga el grado de secundaria; sin embargo, se presentan disimilitudes sustantivas en la tasa de retorno de los distintos etapas del proceso educativo (primaria, superior técnica y universitaria).

El Banco Mundial (2001) estudió la situación de pobreza en Guatemala, teniendo como referente el Informe sobre el desarrollo mundial del Banco Mundial (wDR, por sus siglas en inglés) de 2000 a 2001. Para reducirla, recomienda crear activos para los pobres como educación, igualmente, mejorar la calidad de ésta, con el objetivo de elevar la eficiencia interna y las tasas de retorno de la educación, entre otros.

Desde el punto de vista microeconómico, Dahlin (2003) utilizó la función minceriana de salarios para determinar el efecto de la educación sobre éstos. Los resultados obtenidos muestran que la educación primaria es la que más contribuye a la expectativa de ingresos en países desarrollados y que las tasas de retorno disminuyen con el nivel de escolaridad y el ingreso per capita del país, siendo éstas más altas en el sector privado.

Urrea et al. (2004) muestran cómo el acceso a determinados niveles educativos se reduce en la medida en que disminuye el nivel de ingreso.

Castellar y Uribe (2003) encuentran que una política económica que se concentre únicamente en controlar la inflación, olvidándose de otras variables macroeconómicas como el desempleo, generan un impacto negativo en los grupos más vulnerables sin trabajo, como son los pobres, quienes se caracterizan por ser los menos educados.

Mora (2003) manifiesta que la rentabilidad de la educación superior es la más alta, insinuando con ello las bajas posibilidades que poseen los pobres de rebasar la línea de pobreza. En esta misma línea argumentativa, el acceso a niveles educativos altos es limitado en la medida en que el ingreso disminuye, que es la situación de la población pobre (Urrea et al., 2004).

Los anteriores argumentos sugieren la relevancia de la puesta en marcha de una política educativa adecuada, con el objeto de reducir los índices de pobreza. Es decir, la disminución de la brecha entre ricos y pobres exige una alta inversión en capital humano, acompañada de programas sociales complementarios que les otor- 
guen a las personas todos los activos que requieran para hacerle frente a las dificultadas de la exclusión social.

\section{Modelo Logit múltiple ordenado}

La econometría tradicional asume que en los modelos formulados la variable dependiente es cuantitativa; sin embargo, existen muchas situaciones en las cuales dicha variable es cualitativa, por ejemplo, poseer o no vivienda, tener empleo o no. En esas situaciones, la variable se codifica mediante dígitos o categorías para indicar en cuál de las opciones se ubica el individuo. Cuando el número de categorías es igual a dos, se dice que el modelo es de respuesta dicotómica, en caso contrario, de respuesta múltiple.

La especificación de los modelos de respuesta múltiple está determinada, primero, por el tipo de función de distribución acumulada utilizada; segundo, si las respuestas de los individuos pueden ordenarse o no y, tercero, por las variables consideradas explicatorias. A partir de lo anterior, los modelos más usados son: el Logit —que utiliza la función de distribución acumulada logística—, el Probit — que usa la función de distribución acumulada normal—y el de valor extremo — que aplica la función de distribución acumulada Gompit.

La elección de uno u otro modelo es arbitraria y su diferencia es fundamentalmente operativa, pues el objetivo primordial es la obtención de la probabilidad de que un individuo se clasifique en una categoría, según el valor de las variables explicativas. En ese sentido, los coeficientes estimados no cuantifican directamente el incremento en la probabilidad, dado un incremento unitario en la correspondiente variable independiente. Sin embargo, el signo de los regresores sí indica la dirección del cambio y, naturalmente, cuanto más alta sea dicha pendiente, mayor será el impacto del cambio en la variable explicativa sobre el cambio en la probabilidad.

\section{Modelos de ecuaciones estructurales}

Muchas técnicas estadísticas se concentran en estudiar la relación de dependencia entre una variable $(\mathrm{Y})$ denominada dependiente, $\mathrm{y}$ otras $\left(\mathrm{X}_{1}, \mathrm{X}_{2}, \mathrm{X}_{3} \ldots, \mathrm{X}_{\mathrm{k}}\right)$ llamadas independientes o explicatorias; como ejemplo de dichas técnicas tenemos diferentes tipos de análisis, como el de regresión múltiple, el discriminante, el factorial, entre otros.

El análisis de la relación de dependencia anterior se da en el contexto de una sola ecuación, es decir, se asume que la relación está dada en un sentido, lo cual 
implica que no se considera la existencia de una relación de dependencia en ambos sentidos, en otras palabras, que $\mathrm{Y}$ se explique por $\mathrm{X}_{1}, \mathrm{X}_{2}, \mathrm{X}_{3} \ldots, \mathrm{X}_{\mathrm{k}}$, pero a su vez, Y por alguna(s) variable(s) independiente(s).

Los modelos de ecuaciones estructurales (MES) — también llamados de análisis de la estructura de la covarianza, análisis de variable latente, análisis de factor confirmatorio y, a menudo, simplemente análisis LISREL (por el nombre de unos de los programas de software más utilizado; Hair et al. 1999) — se caracterizan porque pueden estimar varias ecuaciones a la vez, las cuales pueden estar interrelacionadas. Ello implica que la variable dependiente en una ecuación puede ser independiente en otra(s) ecuación(es).

El hecho de que los MEs permitan modelar relaciones más complejas, además de contar con la capacidad de representar conceptos no observados en dichas relaciones, teniendo en cuenta el error de medida en el proceso de estimación, convierten dichos modelos en una herramienta importante para el desarrollo de la investigación aplicada.

Un punto importante por resaltar es que los modelos de ecuaciones estructurales se fundamentan en relaciones causales, en las cuales se asume que una variable origina cambios en otra u otras variables, que es precisamente lo planteado en los modelos de regresión múltiple. No obstante la precisión anterior, es importante tener claridad en cuanto a que la afirmación de causalidad entre dos variables o más depende básicamente de la existencia teórica de dicha relación y no de las técnicas o métodos analíticos elegidos por el investigador.

Los modelos de ecuaciones estructurales siguen una metodología que pasa por las siguientes etapas: especificación, identificación, estimación de parámetros, evaluación del ajuste, reespecificación del modelo e interpretación de los resultados.

\section{Especificación del modelo}

En esta etapa se especifica el modelo estructural, el cual puede tener fines confirmatorios si el objetivo es evaluar la significancia de los parámetros del mismo; fines de confrontación, si el objetivo es decidir entre un conjunto de modelos rivales que representan distintas relaciones hipotéticas estructurales, o fines de búsqueda del modelo adecuado, si el objetivo es desarrollar un modelo que se ajuste adecuadamente a la teoría, partiendo de un modelo inicial, el cual se va reespecificando hasta alcanzar el modelo final. 


\section{Identificación del modelo}

Definido el modelo, se comprueba si es identificable, es decir, si los coeficientes estructurales se pueden estimar. Hay dos normas o reglas básicas para determinar si las ecuaciones de un modelo estructural son identificables o no: condiciones de orden y de rango. Las primeras afirman (Hair et al., 1999) que los grados de libertad del modelo deben ser mayores o iguales a cero. Cuando lo son, el modelo es identificado y cuando es mayor que cero es sobreidentificado; en este último tipo se concentra el interés de los MEs, pues ello implica que se tiene mayor información en la matriz de datos que el número de parámetros por estimar.

Es importante resaltar que la obtención de resultados sin sentido o ilógicos es un síntoma de problemas de identificación, causado, entre otras cosas, por el uso de efectos recíprocos (flechas causales de dos sentidos entre dos constructos). La única solución a un problema de identificación es definir más restricciones para el modelo, es decir, eliminar algunas variables problemáticas.

\section{Etapa de estimación del modelo}

Definida la identificación del modelo, se procede al cálculo de los coeficientes estructurales, el cual se realiza mediante procedimientos iterativos de minimización de desviaciones. Los métodos de estimación utilizados según la estructura de matriz de varianzas y covarianzas son máxima verosimilitud —es un método eficiente y no sesgado cuando se cumplen los supuestos de normalidad multivariada-, y cuando no, se usan, entre otros, el método de mínimos cuadrados ponderados, mínimos cuadrados no ponderados o método de distribución libre asintótica —el cual ha recibido recientemente atención particular debido a su insensibilidad a la no normalidad de los datos.

\section{Evaluación del modelo}

Está asociada a cómo se ajusta al fenómeno de estudio; la calidad de dicho ajuste debe medirse en diferentes niveles: en primer lugar, en el global y, en segundo, en el de cada ecuación que conforma al primero.

Existen diferentes medidas que permiten calcular esa calidad del ajuste, las cuales pueden agruparse en absolutas, de ajuste incremental y de ajuste de parsimonia.

Las medidas absolutas de ajuste se abocan al ajuste del modelo global y entre las más utilizadas están: a) La razón de verosimilitud de la chi-cuadrado $\left(\chi^{2}\right)$, la 
hipótesis nula que indica si el modelo es bueno; cuanto mayor sea el valor obtenido del estadístico $\chi^{2}$, en comparación con los grados de libertad, peor será el ajuste. Esta medida tiende a rechazar frecuentemente cualquier modelo especificado con una muestra suficientemente grande. b) El índice de la bondad de ajuste (goodness of fit index, GFI, por sus siglas en inglés) es una medida no estadística que oscila entre 0 y 1 ; en la medida en que el indicador se aproxime a 1, mejor es el ajuste. $c$ ) El error de aproximación cuadrático medio (root mean square error aproximation, RMSEA, por sus siglas en inglés) procura corregir el defecto de la razón de verosimilitud de la chi-cuadrado $\left(\chi^{2}\right)$; valores de RMSEA inferiores a 0.05 son aceptables.

Las medidas de ajuste incremental evalúan un modelo base o nulo con el modelo propuesto entre las cuales encontramos: $a$ ) El índice ajustado de bondad de ajuste (adjusted goodness of fit index, AGFI, por sus siglas en inglés) representa el GFI ajustado por la razón entre los grados de libertad del modelo propuesto y los del modelo base o nulo; se recomiendan valores mayores o iguales a 0.90 para este indicador. b) El índice de ajuste no normado (non-normed fit index, NNFI, por sus siglas en inglés) combina una medida de parsimonia en un índice comparativo entre los modelos nulos y propuestos; al igual que el AGFI, los valores recomendados como aceptables para este indicador. $c$ ) El índice de ajuste normado (NFI, por sus siglas en inglés) efectúa una comparación en términos relativos entre el modelo propuesto y el modelo nulo; los valores aceptables para este indicador son aquellos superiores o iguales a 0.90 . $d$ ) Existen otras medidas de ajuste incremental, como el índice de ajuste relativo (relative fit index, RFI, por sus siglas en inglés) y el índice de ajuste incremental (incremental fit index, IFI, por sus siglas en inglés), medidas que, como las anteriores, comparan el modelo propuesto con el nulo; si esos índices se acercan a uno, indica que la calidad del ajuste es buena.

Las medidas de ajuste de parsimonia comparan modelos con diferente número de coeficientes para determinar la cantidad de ajuste conseguido al incrementar los coeficientes estimados, entre los más usados están: $a$ ) El índice de ajuste normado (parsimonius normed fit index, PNFI, por sus siglas en inglés), el cual se considera una modificación del NFI, indicador ya comentado. El PNFI tiene en cuenta el número de grados de libertad utilizados para conseguir un nivel de ajuste. Cuando se comparan modelos, se proponen diferencias de 0.06 a 0.09 que sean indicativas de diferencias sustanciales entre los modelos (Hair et al., 1999). b) El índice de calidad de ajuste de parsimonia (parsimonius goodness of fit index, PGFI, por sus siglas en inglés) también se considera una modificación del GFI. El PGFI varía entre 0 y 1 ; valores próximos a 1 indican mayor parsimonia. c) Criterio de información de 
Akaike (akike information criteria, AIC, por sus siglas en inglés): básicamente toma en cuenta el número de constructos que componen los modelos que se pretenden comparar; en la medida en que el AIC se aproxime a 0 , indica que existe mejor ajuste y mayor parsimonia.

Por último, para complementar esta aproximación empírica a la relación entre educación y pobreza, se realiza una comparación de los resultados del MES con los de un modelo Logit de elección múltiple ordenado. Éste permite capturar de forma aislada la misma relación, sin tener presente la interrelación de las variables (Cabrer, Sancho y Serrano, 2001:173-202).

\section{Metodología}

Se parte de la teoría del capital humano y la definición de pobreza desde una concepción de ingreso. En la parte empírica se hace uso de diferentes técnicas cuantitativas, como las tablas cruzadas y los modelos causales propios de la estadística multivariada.

En el contexto de los modelos estimados, las variables empleadas son las siguientes: ESTUD (educación, medida en niveles educativos), POBRE (pobreza, medida en escala ordinal a partir de las líneas de pobreza e indigencia), TAMHOGA (tamaño del hogar, medido en cantidad de personas) y EDAD (medida en años cumplidos).

Para el análisis empírico se procede de la siguiente manera:

a) Se seleccionan las personas ocupadas, así como su nivel educativo, a partir de los módulos de ocupados y de educación de la Encuesta de Calidad de Vida, 2003, del Departamento Administrativo Nacional de Estadística (DANE ECV2003) para el Valle del Cauca. En dicha selección, se excluyen aquellos trabajadores familiares sin remuneración.

b) Los ingresos de las personas ocupadas se construyen a partir de los diferentes rubros presentes en la ECV2003. ${ }^{3}$

c) De acuerdo con el nivel de ingreso de las personas ocupadas calculado en el punto anterior, éstas se clasificaron como indigentes, pobres y no pobres, tomando como referencia la línea de pobreza e indigencia proporcionada por el Departamento Nacional de Planeación. En este trabajo, el ingreso no se ajustó a cuentas nacionales y sólo se emplearon los datos muestrales sin expandir.

3 Esta metodología fue la seguida por la Contraloría General de la República (2004). 
d) Previo a la formulación y estimación del modelo de ecuaciones estructurales, para analizar la causalidad entre los niveles de pobreza y los años de educación, se procede a estimar un modelo Logit de elección múltiple ordenado, con el objeto de contrastar los resultados de un modelo que no incluye la interdependencia entre las variables y el MEs, que sí la incluye.

e) Comparación entre las estimaciones del modelo de ecuaciones estructurales con las de un modelo Logit de elección múltiple ordenado.

f) Elaboración de un cuadro síntesis para analizar estadísticamente la relación educación-pobreza.

g) Elaboración de un cuadro para analizar estadísticamente la relación entre desocupados y el nivel educativo, lo que permite explorar descriptivamente el proceso mediante el cual la educación contribuye a cambiar de la condición de pobre a no pobre.

\section{Análisis descriptivo y modelación}

\section{Análisis descriptivo}

Los cuadros del 1 al 3 permiten adelantar un primer análisis exploratorio de los datos para revisar su consistencia y tener una visión general de su comportamiento.

El cuadro 1 muestra que la mayoría de las personas ocupadas (82.6\%) tiene niveles educativos que no superan la secundaria básica lo cual implica que solamente $17.4 \%$ de la población considerada en la muestra tiene acceso a niveles de educación tecnológica o superior. Por otro lado, se observa que $23.4 \%$ de la población ocupada se ubica por debajo de la línea de pobreza, mientras que $25 \%$, por debajo de la línea de indigencia.

El cuadro 2 presenta la distribución del grado de pobreza para cada uno de los niveles educativos considerados en la población ocupada. En éste se observa que más de $84 \%$ de los encuestados con estudios de educación superior (incompletos o completos) se clasifican como no pobres. En contraposición, más de $60 \%$ de los encuestados sin educación o sólo con la primaria se ubican en la categoría de indigentes o pobres.

El cuadro 3 permite conocer la distribución de los niveles educativos de la población ocupada por grado de pobreza. Los que concentran la población pobre e indigente son de primaria y secundaria, con $84.3 \%$ y $84.6 \%$, respectivamente. Esta situación quiere decir que su acceso a niveles de educación superior es nulo o muy bajo. 
Cuadro 1

Valle del Cauca 2003

Distribución de la población ocupada según los niveles educativo y de pobreza

\begin{tabular}{|c|c|c|c|c|c|c|c|c|c|c|}
\hline \multicolumn{11}{|c|}{ Nivel educativo } \\
\hline $\begin{array}{l}\text { Ingreso } \\
\text { reportado y la } \\
\text { línea de pobreza }\end{array}$ & Ninguno & Primaria & Secundaria & Tecnológico & $\begin{array}{l}\text { Universi- } \\
\quad \text { tario } \\
\text { incompleto }\end{array}$ & $\begin{array}{l}\text { Universi- } \\
\text { tario } \\
\text { completo }\end{array}$ & $\begin{array}{c}\text { Posgrado } \\
\text { incompleto }\end{array}$ & $\begin{array}{l}\text { Posgrado } \\
\text { completo }\end{array}$ & Total & $\begin{array}{c}\text { \% Columna } \\
\text { total }\end{array}$ \\
\hline Indigencia & 55 & 263 & 227 & 19 & 9 & 6 & 0 & 0 & 579 & $25.0 \%$ \\
\hline Pobreza & 47 & 253 & 204 & 20 & 7 & 10 & 1 & 0 & 542 & $23.4 \%$ \\
\hline No pobres & 56 & 346 & 464 & 101 & 88 & 98 & 7 & 38 & 1198 & $51.7 \%$ \\
\hline Total & 158 & 862 & 895 & 140 & 104 & 114 & 8 & 38 & 2319 & $100 \%$ \\
\hline$\%$ fila total & $6.8 \%$ & $37.2 \%$ & $38.6 \%$ & $6.0 \%$ & $4.5 \%$ & $4.9 \%$ & $0.3 \%$ & $1.6 \%$ & $100 \%$ & \\
\hline
\end{tabular}

Fuente: cálculos de los autores con base en ECV 2003 DANE.

Cuadro 2

Valle del Cauca 2003: distribución de la población ocupada de la muestra según nivel educativo y nivel de pobreza (\% verticales)

\begin{tabular}{|c|c|c|c|c|c|c|c|c|}
\hline \multicolumn{9}{|c|}{ Nivel educativo } \\
\hline $\begin{array}{l}\text { Ingreso } \\
\text { reportado y la } \\
\text { línea de pobreza }\end{array}$ & Ninguno & Primaria & Secundaria & Tecnológico & $\begin{array}{l}\text { Universi- } \\
\text { tario } \\
\text { incompleto }\end{array}$ & $\begin{array}{l}\text { Universi- } \\
\text { tario } \\
\text { completo }\end{array}$ & $\begin{array}{l}\text { Posgrado } \\
\text { incompleto }\end{array}$ & $\begin{array}{l}\text { Posgrado } \\
\text { completo }\end{array}$ \\
\hline Indigencia & $34.8 \%$ & $30.5 \%$ & $25.4 \%$ & $13.6 \%$ & $8.7 \%$ & $5.3 \%$ & $0.0 \%$ & $0.0 \%$ \\
\hline Pobreza & $29.7 \%$ & $29.4 \%$ & $22.8 \%$ & $14.3 \%$ & $6.7 \%$ & $8.8 \%$ & $12.5 \%$ & $0.0 \%$ \\
\hline No pobres & $35.4 \%$ & $40.1 \%$ & $51.8 \%$ & $72.1 \%$ & $84.6 \%$ & $86.0 \%$ & $87.5 \%$ & $100.0 \%$ \\
\hline Total & $100 \%$ & $100 \%$ & $100 \%$ & $100 \%$ & $100 \%$ & $100 \%$ & $100 \%$ & $100 \%$ \\
\hline
\end{tabular}

Fuente: cálculos de los autores con base en ECV 2003 DANE.

Cuadro 3

Valle del Cauca 2003: distribución de la población ocupada de la muestra según nivel educativo y nivel de pobreza (\% horizontales)

\begin{tabular}{|c|c|c|c|c|c|c|c|c|c|}
\hline \multicolumn{10}{|c|}{ Nivel educativo } \\
\hline $\begin{array}{l}\text { Ingreso } \\
\text { reportado y la } \\
\text { línea de pobreza }\end{array}$ & Ninguno & Primaria & Secundaria & Tecnológico & $\begin{array}{l}\text { Universi- } \\
\text { tario } \\
\text { incompleto }\end{array}$ & $\begin{array}{l}\text { Universi- } \\
\text { tario } \\
\text { completo }\end{array}$ & $\begin{array}{c}\text { Posgrado } \\
\text { incompleto }\end{array}$ & $\begin{array}{l}\text { Posgrado } \\
\text { completo }\end{array}$ & Total \\
\hline Indigencia & $9.5 \%$ & $45.4 \%$ & $39.2 \%$ & $3.3 \%$ & $1.6 \%$ & $1.0 \%$ & $0.0 \%$ & $0.0 \%$ & $100 \%$ \\
\hline Pobreza & $8.7 \%$ & $46.7 \%$ & $37.6 \%$ & $3.7 \%$ & $1.3 \%$ & $1.8 \%$ & $0.2 \%$ & $0.0 \%$ & $100 \%$ \\
\hline No pobres & $4.7 \%$ & $28.9 \%$ & $38.7 \%$ & $8.4 \%$ & $7.3 \%$ & $8.2 \%$ & $0.6 \%$ & $3.2 \%$ & $100 \%$ \\
\hline
\end{tabular}

Fuente: cálculos de los autores con base en ECV 2003 DANE. 


\section{Modelación}

\section{Modelo Logit múltiple ordenado}

Dado que las variables dependientes, en nuestro caso pobreza y educación, pueden tomar diferentes categorías y, a su vez, pueden ser ordenadas (niveles de educación y pobreza), se emplea un modelo Logit múltiple ordenado (Cabrer, Sancho y Serrano 2001:173-174).

Los resultados que se presentan primero son para analizar la relación entre educación y pobreza (cuadro 4) y, además, muestran que los signos de las variables independientes o explicatorias son teóricamente consistentes, pues a medida que se avanza de la condición de pobre a no pobre (POBRE), el nivel educativo (ESTUD) aumenta (signo positivo y estadísticamente significativo). El estadístico de razón de verosimilitud, que prueba si el modelo planteado es adecuado, muestra que se rechaza la hipótesis de no significancia, indicando con ello que el modelo propuesto es adecuado.

\section{Cuadro 4}

\begin{tabular}{|c|c|c|c|c|}
\hline $\begin{array}{l}\text { Variable dependiente: ESTUD } \\
\text { Método: ML - Logit ordenad } \\
\text { Fecha: 15/12/2006 Tiempo: } \\
\text { Muestra: } 12319 \\
\text { Observaciones incluidas: } 2 \\
\text { Número de valores de indic } \\
\text { Convergencia obtenida a pa } \\
\text { Matriz de covarianza calcul }\end{array}$ & $\begin{array}{l}0 \\
5 \text { iteraciones } \\
\text { partir de las segu }\end{array}$ & ndas derivadas & & \\
\hline Variables & Coeficientes & Error estándar & Estadístico Z & Prob. \\
\hline $\begin{array}{l}\text { POBRE } \\
\text { EDAD }\end{array}$ & $\begin{array}{r}0.731897 \\
-0.042786\end{array}$ & $\begin{array}{l}0.049162 \\
0.002806\end{array}$ & $\begin{array}{r}14.88739 \\
-15.24784\end{array}$ & $\begin{array}{l}0 \\
0\end{array}$ \\
\hline $\begin{array}{l}\text { LIMIT_2:C(3) } \\
\text { LIMIT_3:C(4) } \\
\text { LIMIT_4:C(5) } \\
\text { LIMIT_5:C(6) } \\
\text { LIMIT_6:C(7) } \\
\text { LIMIT_7:C }(8) \\
\text { LIMIT_8:C(9) }\end{array}$ & $\begin{array}{l}\text { Puntos límites } \\
-2.902913 \\
-0.234187 \\
1.785909 \\
2.31507 \\
2.884374 \\
4.193286 \\
4.387931\end{array}$ & $\begin{array}{l}0.163636 \\
0.140181 \\
0.146165 \\
0.151457 \\
0.160107 \\
0.20349 \\
0.214497\end{array}$ & $\begin{array}{l}-17.74004 \\
-1.670596 \\
12.21846 \\
15.28535 \\
18.01528 \\
20.60684 \\
20.45679\end{array}$ & $\begin{array}{l}0 \\
0.0948 \\
0 \\
0 \\
0 \\
0 \\
0\end{array}$ \\
\hline $\begin{array}{l}\text { Criterio de infor. de Akaike } \\
\text { Logaritmo de probab. } \\
\text { Restr. logaritmo de probab. } \\
\text { LR estadístico ( } 2 \text { df) } \\
\text { Probabilidad (LR stat) }\end{array}$ & $\begin{array}{r}2.759235 \\
-3190.333 \\
-3390.517 \\
400.3666 \\
0\end{array}$ & $\begin{array}{l}\text { Criterio de Schw } \\
\text { Criterio de Hann } \\
\text { Prom. del logaritn } \\
\text { LR índice (Pseud }\end{array}$ & $\begin{array}{l}\text { z } \\
\text { n-Quinn } \\
\text { de probab. } \\
-\mathrm{R} 2)\end{array}$ & $\begin{array}{r}2.781547 \\
2.767366 \\
-1.375737 \\
0.059042\end{array}$ \\
\hline
\end{tabular}

Fuente: cálculos propios. 
Cuadro 5

Variable dependiente: POBRE

Método: ML - Logit ordenado

Fecha: 15/12/2006 Tiempo: 10:21

Muestra: 12319

Observaciones incluidas: 2319

Número de valores de indicadores ordenados: 3

Convergencia obtenida a partir de 4 iteraciones

Matriz de covarianza calculada a partir de las segundas derivadas

\begin{tabular}{lrrrr}
\multicolumn{1}{c}{ Variables } & Coeficientes & Error estándar & Estadístico Z & Prob. \\
ESTUD & 0.442849 & 0.033550 & 13.19959 & 0.0000 \\
TAMHOGA & -0.152390 & 0.033113 & -4.602075 & 0.0000 \\
& \multicolumn{2}{c}{ Puntos Límites } & & \\
& -0.313143 & 0.122329 & -2.559831 & 0.0105 \\
LIMIT_2:C(3) & 0.797656 & 0.122481 & 6.512476 & 0.0000 \\
LIMIT_3:C(4) & 1.960449 & Criterio de Schwarz & 1.970365 \\
Criterio de infor. de Akaike & -2269.141 & Criterio de Hannan-Quinn & 1.964063 \\
Logaritmo de probab. & -2382.537 & Prom. del logaritmo de probab. & -0.978500 \\
Restr. logaritmo de probab. & 226.7920 & LR índice (Pseudo-R2) & 0.047595 \\
LR estadístico (2 df) & 0.000000 & & \\
Probabilidad (LR stat) & & &
\end{tabular}

Fuente: cálculos propios.

En el cuadro 5 (relación de pobreza y educación) se muestra que las variables explicatorias son estadísticamente significativas: los signos son consistentes con la teoría, pues a medida que se avanza en el nivel educativo (ESTUD), se pasa de condición de pobre a no pobre (POBRE), además se encuentra que el aumento del tamaño del hogar (TAMHOGA) está asociado a hogares más pobres, resultado consistente con la mayoría de estudios realizados en esa dirección. El estadístico de razón de verosimilitud indica que el modelo propuesto es adecuado.

Los resultados anteriores se hacen en un contexto uniecuacional, sin tener en cuenta la interrelación de las variables, la cual se pretende analizar con los modelos estructurales, planteados y estimados a continuación.

Las ecuaciones estructurales propuestas para el modelo fueron las siguientes:

$$
\begin{aligned}
& \text { ESTUD }=\gamma_{10}+\gamma_{11} \text { EDAD }+\gamma_{12} \text { POBRE }+\varepsilon_{1} \\
& \text { POBRE }=\gamma_{20}+\gamma_{21} \text { TAMHOGA }+\gamma_{22} \text { Estud }+\varepsilon_{2}
\end{aligned}
$$

Entre los criterios para identificar el modelo, podemos referir que las condiciones de orden y rango se cumplan (Hair et al., 1999), el software las valida 
automáticamente, pero se puede apreciar de manera clara que estas ecuaciones son identificables según las condiciones de orden. La gráfica 1 muestra el modelo estructural formulado, donde los rectángulos representan las variables observables o atributos; las líneas rectas que salen de los rectángulos indican los efectos directos; las líneas rectas horizontales de izquierda a derecha, el error de medida; la línea curvada que une los rectángulos, la correlación existente entre las variables exógenas. Se debe tener en cuenta que en la gráfica el modelo está asumiendo una relación en ambos sentidos, entre las variables pobreza y educación.

$\mathrm{Al}$ estimar el modelo propuesto, se obtiene un ajuste del modelo global adecuado pues el RMSEA es inferior de $0.05\left(\mathrm{H}_{0}\right.$ : el ajuste del modelo es adecuado). Para la interpretación de los resultados de los modelos hay que tener presente en adelante que los valores entre paréntesis corresponden a los errores estándar y los que aparecen con éstos son los estadísticos $t$.

\section{Salida de LISREL mínimos cuadrados ponderados (weighted least squares)}

$\begin{array}{rrrr}\text { ESTUD }= & 28.25+ & 0.65{ }^{*} \text { POBRE }-0.51 * \text { EDAD, Errorvar. }= & 0.75, \mathrm{R}^{2}=0.25 \\ (30.44) & (0.62) & (0.80) & (1.21) \\ 0.93 & 1.05 & -0.64 & 0.62 \\ \text { POBRE }= & & & \\ 3.85- & 0.41{ }^{*} \text { ESTUD }-0.11 * \text { TAMHOGA, Errorvar. }= & 1.41, \mathrm{R}^{2}=-0.41 \\ (7.72) & (0.86) & (0.056) & (1.24) \\ 0.50 & -0.47 & -1.92 & 1.13\end{array}$

Sin embargo, al evaluar en detalle cada una de las variables, se puede apreciar que ninguna es estadísticamente significativa y que hay presencia de signos erróneos en los coeficientes, tanto en la variable educación (ESTUD) como en el $\mathrm{R}^{2}$ de la ecuación de pobreza. Pues teóricamente se espera que el efecto de la educación en la pobreza sea positivo, es decir, que a medida que la persona tiene más años de estudio, sus ingresos mejoren y, por consiguiente, que sea menos pobre. Lo anterior sugiere la existencia de estimaciones infractoras, lo cual plantea la necesidad de reespecificar la estructura del modelo original para mejorar sus resultados.

Si se toma en cuenta que una posible causa para obtener los resultados anteriores tan ilógicos y sin sentido es el uso de efectos recíprocos (flechas causales de dos sentidos entre dos constructos), entonces se decide eliminar en la nueva especificación la variable pobreza como determinante de los años de estudio, pues éstos son los que determinan el nivel de aquélla. La gráfica 2 muestra el nuevo modelo considerado. 

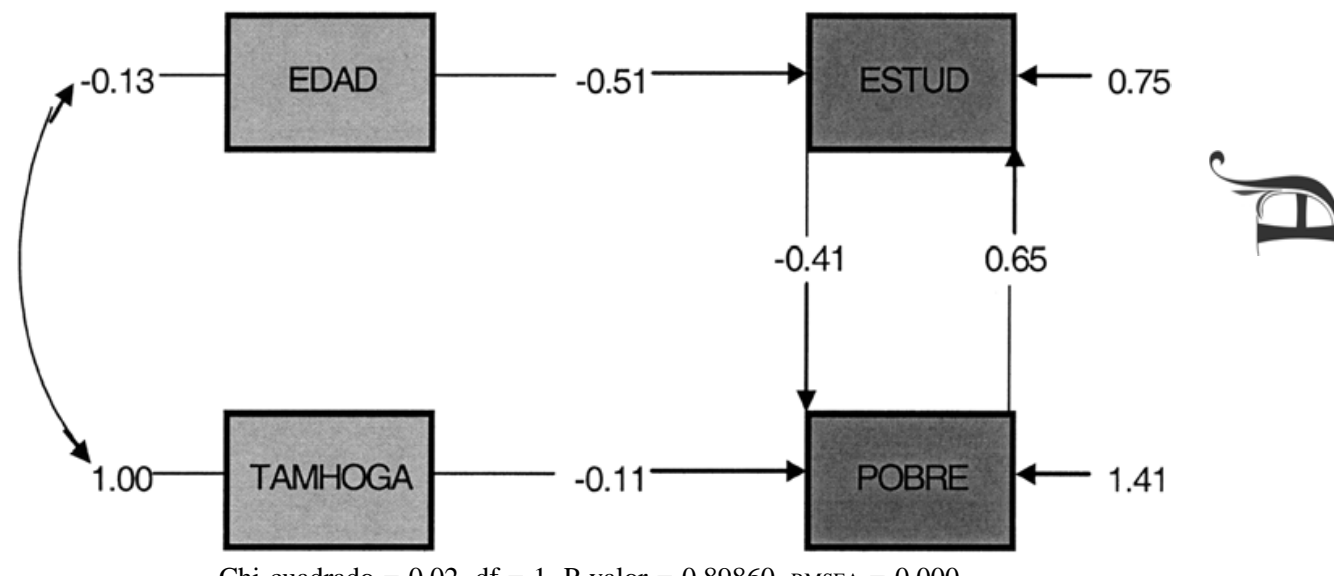

Gráfica 1.

Fuente: elaborado por los autores con base en estimaciones del modelo MES.

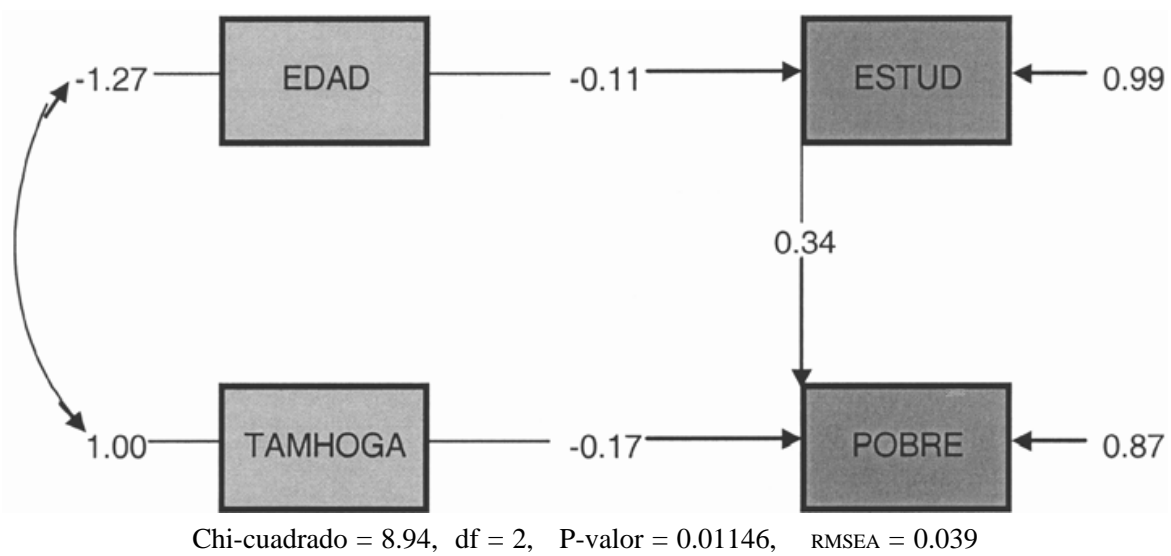

Gráfica 2.

Fuente: elaborado por los autores con base en estimaciones del modelo MEs.

La estimación es la siguiente:

Salida de LISREL mínimos cuadrados ponderados (weighted least squares)

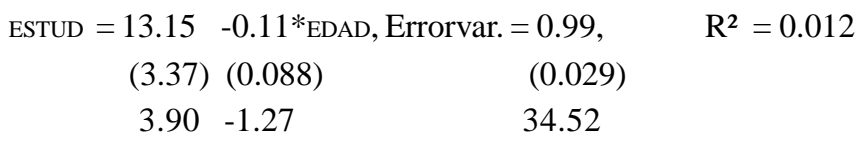


POBRE $=-2.63+0.34 *^{*}$ ESTUD $-0.17 *^{*}$ TAMHOGA, Errorvar. $=0.87, \quad \mathrm{R}^{2}=0.13$
(0.21) (0.029)
$(0.035)$
(0.026)
$-12.4911 .71$
$-4.79$
33.09

Al eliminar el sendero de pobreza a educación, el modelo global arroja un RMSEA menor que 0.05 , lo cual sugiere un buen ajuste. Se destaca en esta estimación tanto la consistencia de signos para los coeficientes de las variables educación (ESTUD) y tamaño del hogar (TAMHOGA) como la significancia de éstas. El signo positivo del coeficiente de educación supone que cuando los años de estudio aumentan, la variable pobreza también, ${ }^{4}$ lo cual implica que se pasa de umbrales de pobreza a los de no pobreza.

El signo negativo del coeficiente de la variable tamaño del hogar sugiere que en la medida en que el tamaño de éste se incrementa, se pasa a umbrales de pobreza, lo cual es consistente con muchos estudios en donde se encuentra que los hogares pobres se caracterizan por estar compuestos por gran cantidad de personas.

Finalmente, se define otro modelo en el cual los años de estudio no explican los niveles de pobreza, pero ésta sí determina los años de estudio. La gráfica 3 muestra el tercer modelo considerado.

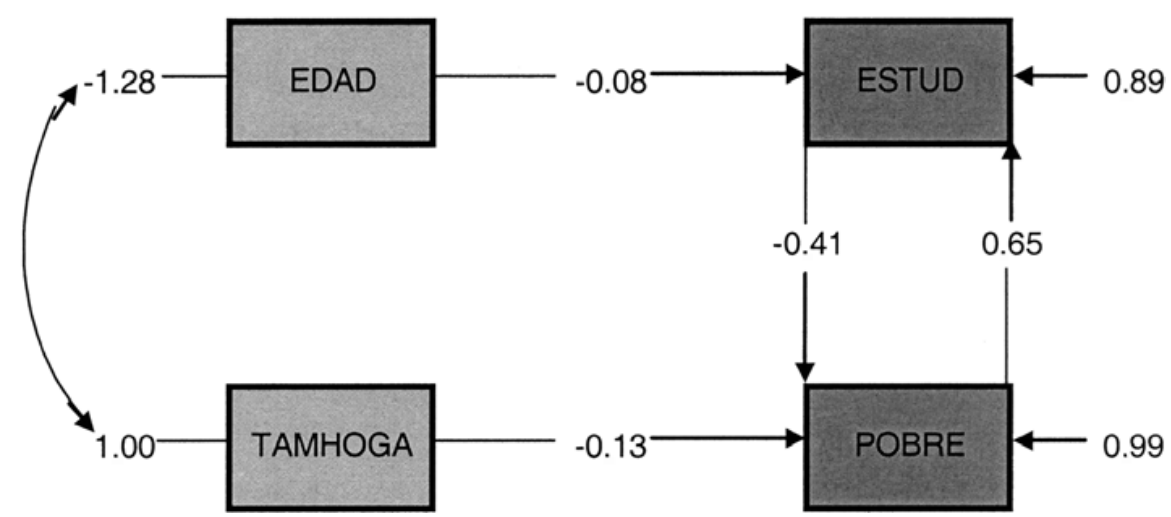

Chi-cuadrado $=10.48, \quad \mathrm{df}=2, \quad \mathrm{P}$-valor $=0.00530$, RMSEA $=0.043$

Gráfica 3.

Fuente: cálculos de los autores con base en estimaciones del MES.

$4 \quad$ Es importante recordar que la pobreza se está midiendo en una escala ordinal 1, 2, 3, donde $1=$ indigencia, 2 = pobreza moderada y $3=$ no pobre, es decir, que a medida que aumente la escala se pasa de estadios de pobreza extrema a no pobreza. 
El resultado de la estimación es el siguiente:

Salida de LISREL mínimos cuadrados ponderados (weighted least squares)

$$
\begin{aligned}
& \text { ESTUD }=15.41+0.19 * \text { POBRE- } 0.14 * \text { EDAD }+ \text { Errorvar. }=0.76, \mathrm{R}^{2}=0.24 \\
& \text { (3.33) (0.021)(0.091) (0.048) } \\
& \begin{array}{ll}
4.63 \quad 8.85-1.54 & 15.74
\end{array} \\
& \text { POBRE }=0.43-0.096 * \text { TAMHOGA, Errorvar. }=0.99, \mathrm{R}^{2}=0.0092 \\
& \text { (0.055) (0.012) (0.018) } \\
& \begin{array}{lll}
7.81 & -8.32 & 55.08
\end{array}
\end{aligned}
$$

Los resultados obtenidos con este tercer modelo arrojan un ajuste global adecuado, pues RMSEA $<0.05$, además, los signos obtenidos son teóricamente consistentes, lo cual sugiere que en la medida en que una persona pasa del umbral de pobre a no pobre, los años de estudio aumentan; de igual manera, el signo del coeficiente de la variable tamaño del hogar en la ecuación 2 es consistente, de acuerdo con lo expuesto anteriormente. Llama la atención el valor del $\mathrm{R}^{2}$ de la ecuación de pobreza, pues aunque el tamaño del hogar es significativo, dicho valor indica que es muy poco lo que ésta aporta para la explicación de la pobreza.

Al comparar los resultados obtenidos por el modelo Logit múltiple ordenado con los del estructural, se deduce que en ambos los signos son consistentes con la teoría, las variables explicatorias son estadísticamente significativas y los modelos planteados son adecuados, según las pruebas utilizadas. Sin embargo, la magnitud de los coeficientes difieren de manera significativa, lo cual puede ser justificado porque el modelo Logit, como se ha mencionado a lo largo del trabajo, no considera la relación existente entre las variables educación y pobreza, mientras el modelo de ecuaciones estructurales sí lo hace.

En resumen, los resultados encontrados con el modelo estructural sugieren que existe un proceso en doble vía entre los niveles de pobreza y de educación, es decir, aquellas personas pobres alcanzan bajos grados de educación, posiblemente por su naturaleza de pobres (medidos por líneas de pobreza) y aquellas personas con bajos niveles de educación son pobres. Lo anterior se ratifica al observar el cuadro 6, el cual contiene los diferentes indicadores de calidad del ajuste.

Finalmente, los resultados encontrados con el modelo 3 de ecuaciones estructurales ratifican la observación realizada al inicio con la información descriptiva de 
Cuadro 6

Evaluación global de los modelos

\begin{tabular}{|c|c|c|c|c|c|}
\hline $\begin{array}{c}\text { Grupo de } \\
\text { indicadores }\end{array}$ & Índice & $\begin{array}{l}\text { Valor de } \\
\text { referencia }\end{array}$ & Modelo 1 & Modelo 2 & Modelo 3 \\
\hline \multirow{5}{*}{ Ajuste absoluto } & & No significativo & 0.02 & 9.14 & 10.80 \\
\hline & $\chi^{2}$ & $(\mathrm{p}>0.05)$ & $(\mathrm{p}=0.898)$ & $(p=0.010)$ & $(\mathrm{p}=0.004)$ \\
\hline & RMSEA & $\leq 0.05$ & 0.00 & 0.039 & 0.044 \\
\hline & GFI & $\geq 0.90$ & 1.00 & 1.00 & 1.00 \\
\hline & AGFI & $\geq 0.90$ & 1.00 & 1.00 & 0.99 \\
\hline \multirow[t]{6}{*}{ Ajuste incremental } & NFI & $\geq 0.90$ & 1.00 & 0.98 & 0.98 \\
\hline & NNFI & $\geq 0.90$ & 1.01 & 0.95 & 0.94 \\
\hline & CFI & $\geq 0.90$ & 1.00 & 0.98 & 0.98 \\
\hline & IFI & $\geq 0.90$ & 1.00 & 0.98 & 0.98 \\
\hline & RFI & $\geq 0.90$ & 1.00 & 0.94 & 0.93 \\
\hline & RMR & $\leq 0.10$ & 0.052 & 0.37 & 0.38 \\
\hline \multirow[t]{3}{*}{ Ajuste de parsimonia } & PGFI & $\geq 0.90$ & 0.100 & 0.20 & 0.20 \\
\hline & PNFI & $(0.6-0.9)$ & 0.170 & 0.33 & 0.33 \\
\hline & AIC & Mín. (AIC) & 26.02 & 33.14 & 34.80 \\
\hline
\end{tabular}

Fuente: cálculos de los autores.

los cuadros 1 al 3 sobre el bajo acceso de la población pobre a los niveles de educación superior, pues al pasar de la categoría de no pobre (3) a la pobre (2) o indigente (1), los años de educación disminuyen.

Una reflexión acerca del proceso mediante

el cual la educación contribuye en la reducción de la pobreza

La educación se ha señalado como una estrategia de política social imprescindible para que el individuo alcance su bienestar mediante el incremento de sus ingresos. Si bien la educación permite alcanzar esa situación, además genera aspectos que son difícilmente medibles, no obstante pueden ser observables. Una sociedad con altos niveles de educación puede permitirse la legitimidad de la noción de orden social, lo que produce un acuerdo entre la organización social y los individuos.

En ciertas circunstancias, los individuos pobres proceden de hogares pobres en los que no se valora la educación, lo cual ha provocado, por un lado, la dificultad en la movilidad educativa intergeneracional, construyendo un individuo poco informado y, por otro lado, la ausencia de legitimidad de la organización, es decir, el reconocimiento del orden social. 
Cuadro 7

Valle del Cauca, 2003

Distribución de la población desocupada

según la ECV2003 por nivel educativo

\begin{tabular}{lcr}
\hline Nivel educativo & Frecuencia & $\%$ \\
\hline Ninguno & 16 & 4.17 \\
Primaria & 121 & 31.51 \\
Secundaria & 184 & 47.92 \\
Tecnológica & 23 & 5.99 \\
Universitaria incompleta & 27 & 7.03 \\
Universitaria completa & 9 & 2.34 \\
Posgrado completo & 4 & 1.04 \\
\hline Total & 384 & 100 \\
\hline
\end{tabular}

Fuente: cálculos de los autores, con base ECv2003 DANE.

Indudablemente, los argumentos expuestos acerca del papel de la educación señalan que por medio de ella se podría superar la pobreza; sin embargo, debe ir acompañado de un marco institucional que brinde condiciones adecuadas para que una vez que los individuos cumplan su proceso formativo, contribuyan económica y políticamente en la conformación de una sociedad incluyente.

En efecto, como se observa en el cuadro 7, existen personas que han finalizado las distintas etapas del proceso formativo, pero, a pesar de ello, no hay condiciones en el mercado de trabajo que les permitan la generación de ingresos, lo cual podría implicar la exclusión social y, por ende, los desequilibrios sociales.

En general, los estudios de tasa de retorno sugieren que la educación primaria expone retornos sociales mayores que la educación superior, pero, al mismo tiempo, consideran que la educación secundaria no es retribuida por el mercado laboral. Ello sugeriría el diseño de la política educativa de largo plazo, que tenga por objeto la vinculación de la población pobre a la educación superior. Esta situación se hace cada vez más necesaria, si se tienen en consideración los procesos de integración de las economías en el contexto de la globalización.

Hoy en día, el acceso a la educación superior es inalcanzable para las personas pobres, debido a los altos costos económicos y sociales. Contrariamente al propósito que tienen las universidades públicas — de facilitar el proceso formativo de la población con menos recursos económicos-, éstas están vinculando estudiantes provenientes de estratos socioeconómicos altos, y con ello generan, por un lado, que no se logre la función equidistributiva de la universidad pública y, por otro, que la brecha educativa y económica entre pobres y no pobres se amplíe. 
La existencia de personas con educación superior desocupadas plantea la necesidad de llevar a cabo investigaciones en las cuales se diluciden más claramente los factores que expliquen dicha situación, pues si bien es cierto que se consideran fundamentales las condiciones macroeconómicas, es posible que existan otras, como la calidad de la educación, cierto tipo de profesiones que no permiten la rápida vinculación al mercado laboral o la no vinculación a un conjunto de redes sociales que faciliten la integración al mercado laboral.

\section{Conclusiones}

Desde el punto de vista de la teoría del capital humano, se sugiere que la educación — por sus efectos sobre la productividad y la generación de ingresos- constituye un instrumento clave en la política de superación de la pobreza. No obstante, pocos estudios empíricos analizan la interrelación de educación y pobreza.

En un contexto uniecuacional, sin tener en cuenta la interrelación de las variables objeto de estudio —niveles de educación y de pobreza—, el modelo Logit múltiple ordenado muestra cómo ambas variables están relacionadas; en la población empleada, los bajos grados de educación explican los altos niveles de pobreza y viceversa.

Profundicemos en el resultado anterior: se analizó la potencial interrelación de las variables; el nivel de pobreza como determinante del nivel educativo y viceversa. Los modelos de ecuaciones estructurales estimados corroboran empíricamente dicha interrelación.

En efecto, existe un proceso en doble vía entre los niveles de pobreza y de educación; es decir, aquellas personas pobres alcanzan bajos grados de educación posiblemente por su naturaleza de pobres (medidos por líneas de pobreza), y aquellas personas con bajos niveles de educación que son pobres.

Una posible salida del círculo vicioso entre educación y pobreza sería subsidiar la educación en todos sus niveles y que todas las personas tengan acceso a la misma. Sin embargo, los resultados muestran que no basta con educar a las personas sólo para superar la pobreza, si no se garantiza un entorno macroeconómico favorable, específicamente en términos de empleo, además de otros factores, los cuales no se abordan en el presente trabajo.

Con base en las anteriores conclusiones, se sugieren las siguientes recomendaciones en términos de política pública.

La política educativa se ha preocupado por garantizar que la educación básica llegue a todas las personas; sin embargo, ese nivel no es suficiente para alterar la 
condición de pobre a no pobre. Los estudios empíricos muestran, en relación con las tasas de retorno privadas de la educación, que el mercado laboral no retribuye la secundaria, pues la considera un paso de transición a la educación superior, y que el mayor retorno se consigue en ésta (Mora, 2003). Ello sugiere que es a dicho nivel educativo al que se debería facilitársele el acceso a la población pobre para que aumenten las posibilidades de superar la condición de pobre en condiciones macroeconómicas favorables.

Finalmente, se presentan, entre otros, dos factores que se convierten en obstáculos para que estudiantes pobres puedan tener acceso a la educación superior. El primero está asociado a la calidad del nivel secundaria que reciben los estudiantes de bajos ingresos, lo cual no permite que sean competitivos por los cupos que se ofrecen en las instituciones públicas de estudios superiores. El segundo factor tiene que ver con los costos asociados a ésta, pues como lo sugieren los resultados encontrados por Urrea et al. (2004), el acceso a grados educativos altos se reduce en la medida en que el nivel de ingreso disminuye, el cual es el caso de los pobres.?

\section{Bibliografía}

Banco Mundial, "Attacking Poverty", Washington, World Development Report (WDR) 2000-2001, 2001.

Barro, Robert, "Economic Growth in a Cross Section of Countries," en Quarterly Journal of Economics, núm 2, vol. 106, Cambridge, мIт Press, mayo, 1991, pp 407-443.

Becker, Gary, Human Capital: A Theoretical and Empirical Analysis, with Special Reference to Education, Chicago, University of Chicago Press, 1993.

, "Investment in Human Capital: A Theoretical Analysis", en Journal of Political Economy, Chicago Press, núm. 5, vol. 70, Chicago, octubre, 1962, pp. 9-49.

Borjas, George, Labor Economics, McGrawHill/Irwin, New York, 1999.

Cabrer, Bernardí, Amparo Sancho y Guadalupe Serrano, Microeconometría y decisión, Madrid, Ediciones Pirámide, 2001.

Castellar, Carlos y José Ignacio Uribe, "La tasa de retorno de la educación: teoría y evidencia micro y macroeconómicas en el área metropolitana de Cali 1988-2000”, en Documento de trabajo núm. 66, CIDSE, Cali, Universidad del Valle, 2003.

Chávez, Álvaro Hernando y Helmuth Yesid Arias, "Cálculo de la tasa interna de retorno de la educación en Colombia", en Documento de trabajo núm. 2, Bogotá, Universidad Externado de Colombia, 2002.

Comisión Económica para América Latina (CEPAL), Naciones Unidas, "Panorama social de América Latina 2004", http://www.cepal.cl/publicaciones/ DesarrolloSocial/0/LCL222 OPE/CapI Pobreza_version_preliminar. pdf, 2005.

, "Invertir mejor para invertir más, Financiamiento y gestión de la educación en América Latina y el Caribe", en Serie Seminarios y Conferencias, núm. 43, 2005.

Contraloría General de la República, "El debate Sistema sobre el tamaño de la pobreza en Colombia", en Economía Colombiana, núm. 303, Contraloría General de la República, Bogotá, julio-agosto de 2004.

Dahlin, Brian G, "The Impact of Education on Economic Growth: Theory, Findings, and Policy Implications", http://www.duke.edu/ 
$\sim$ bgd3/bgd0202.pdf, Durham NC, Duke University, 2003.

Departamento Administrativo Nacional de Estadística (DANE), "Encuesta de Calidad de Vida 2003, Bogotá, Dirección de Metodología y Producción Estadística, marzo de 2003.

De la Fuente, Ángel, "Educación y crecimiento: un panorama", en Revista Asturiana de Economía, núm. 31, Oviedo, 2004.

DNP, PNUD, PNDH Y GTZ, Los municipios colombianos hacia los objetivos de desarrollo del milenio: salud, educación y reducción de la pobreza, Bogotá, 2006.

Easterly, William, En busca del crecimiento, andanzas y tribulaciones de los economistas del desarrollo, Barcelona, Antoni Bosch Editor, 2003.

González, Francisco, Carolina Guzmán y Ángela Pachón, "Productividad y retornos sociales del capital humano: microfundamentos y evidencia para Colombia", en Archivos de macroeconomía, núm. 98, Bogotá, Departamento Nacional de Planeación, 1998.

Giménez, Gregorio, "La dotación de capital humano de América Latina y el Caribe", en Revista de la CEPAL, núm. 86, 2005, pp, 103-122.

Hair, Joseph, Rolph Anderson, Ronald Tatham y William Black, Análisis multivariante, Prentice Hall, Madrid, 1999.

Johnes, Geraint, Economía de la Educación, Capital humano, rendimiento educativo y mercado de trabajo, Barcelona, Ministerio de trabajo, Editorial Ariel, 1995.

Mora, Jhon James, "Las ganancias de tener un título: una aplicación al mercado laboral de Cali”, Lecturas de Economía, núm. 59, Medellín, Universidad de Antioquia, 2003, pp. 55-72.

Millán, Natalia, Perfil de la pobreza en la Costa Atlántica, Misión para el diseño de una estrategia para la reducción de la pobreza y la desigualdad (MERPD), 2005.

Núñez, Jairo, Determinantes de la pobreza en Colombia, 1996-2004, Documento CEDE, 2005-60, Issn 1657-7191, Bogotá, Universidad de los Andes, 2005.

- y Silvia Espinosa, Determinantes de la Pobreza y la Vulnerabilidad, Bogotá, Documento de la Misión para el Diseño de una Estrategia para la Reducción de la Pobreza y la Desigualdad (MERPD), Departamento Nacional de Planeación (DNP), 2005.
Organización de Naciones Unidas (ONU), Declaración del Milenio, resolución A/RES/ 55/2 aprobada por la Asamblea General en su quincuagésimo quinto periodo de sesiones, Naciones Unidas, septiembre de 2000, http://www.un.org/spanish/millennium goals/index.html.

Pardo, Oliver, "Acumulación de capital humano y gasto público en educación: Un Modelo OLg para Colombia", Archivos de Macroeconomía, núm. 303, Bogotá, Departamento Nacional de Planeación, 2006.

Ramos, Virgilio, "La Educación y la Circularidad de la Pobreza", Lima, enero de 2000, http:// www,inei,gob,pe/biblioineipub/ban copub/ Est/Lib0079/INTRO,htm.

Ravallion, Martin "Poverty Comparisons, A Guide to Concepts and Methods", Living Standards Measurement Study Working Paper, núm, 88, Washington DC, The Word Bank, 1992, pp. 1-138.

Ravallion, Martin, "Poverty Line Theory and Practice", Living Standards Measurement Study Working Paper, núm. 133, 1998, pp. 1-53.

Sala-i-Martin, Xavier, " 15 years of New Growth Economics: What Have We Learnt?", en Documentos de trabajo, núm. 172, Santiago de Chile, Banco Central de Chile, 2001.

Shack, Nelson, La Pobreza, la desigualdad y la educación en el Perú de hoy: una aproximación cuantitativa, Santiago de Chile, Universidad de Chile, 1999.

Sánchez, Fabio, Jorge Rodríguez y Jairo Núñez, "Evolución y determinantes de la productividad en Colombia: un análisis global y sectorial", Archivos de macroeconomía, núm. 50, Bogotá, Departamento Nacional de Planeación, 1996.

Sánchez, Fabio y Jairo Núñez, "Educación y salarios relativos en Colombia: Determinantes y Evolución: 1976-1995 Determinantes, evolución e implicaciones para la distribución del ingreso", Archivos de Macroeconomía, núm. 74, Bogotá, De-partamento Nacional de Planeación, 1998.

Urrea, Fernando, Jaime Escobar, Inés Ulloa y Juan Correa, Condiciones sociodemográficas, pobreza y desigualdad en el Valle del Cauca: un análisis basado en la Encuesta de Calidad de Vida DANE 2003, Cali, Grupo de Investigación sobre Pobreza y Desigualdad, Informe final, CIDSE-Universidad del Valle, 2003. 